\title{
Evaluation of the Low Cost Telemedicine System in Taiwan
}

\author{
J.J. Luh, P.H. Cheng* ${ }^{*}{ }^{\dagger}$, S.J. Chen*, J.S. Lai ${ }^{\ddagger}$, H.S. Chen** and H.C. Chung ${ }^{\dagger}$ \\ School and Graduate Institute of Physical Therapy, College of Medicine, \\ National Taiwan University, Taipei, Taiwan \\ * Graduate Institute of Electronics Engineering and Department of Electrical Engineering, \\ National Taiwan University, Taipei, Taiwan \\ $\dagger$ Department of Information Systems, \\ National Taiwan University Hospital and College of Medicine, \\ National Taiwan University, Taipei, Taiwan \\ ‡Department of Physical Medicine and Rehabilitation, College of Medicine, \\ National Taiwan University, Taipei, Taiwan \\ **Departments of Family Medicine and Medical Informatics, \\ National Taiwan University, Taipei, Taiwan \\ jslai@ccms.ntu.edu.tw
}

\begin{abstract}
A low cost telemedicine system which constructed with inexpensive, commercially available parts and used ADSL network was developed for tele-consultation. This system enhanced the acceptability of the healthcare services for the mountainous region and redeem the lack of medicine man power in such area. The preliminary results from several medical centers and local hospital show that this telemedicine system can meet the requirement of telemedicine uses.
\end{abstract}

\section{Introduction}

From 1992, the Cyber-Care Inc.'s Electronic HouseCall (EHC) system could connect the blood pressure monitor, glucose monitor, digital thermometer, digital stethoscope, arterior oximeter, and weight scale. Currently, NTUH uses EHC-350 and Li-Shin tribe uses EHC-400. Both of them are connected with ISDN lines[1][2][3]. We found some existing problems listed as follows:

- In order to pursue the accuracy of clinic diagnosis, obviously, we have to invest a high budget on the related telemedicine equipments.

- The system usage frequency is obviously very low.

- Instability of the communication quality implicitly hides behind the whole system.

- The communication fee is so high that no one likes to invest on the system any more.

- If the government stops the budget support, the routine telemedicine cannot be continued.

- The health insurance payment is not enough to cover the operating cost.

- It is very difficult to evaluate the ratio of the return on investment.

Therefore, a low cost telemedicine should be designed to solve the problems mentioned above. In this study, we tried to design a telemedicine and evaluate its performance according by questionnaire.

\section{Materials and Methods}

We cooperated with two national medical centers, two regional hospitals, and five remote native tribes (Fu-San, Li-Shin, Gin-Gun, Won-Fong, and Xai-Nun) which are all located inside Taiwan Central Mountain. This telemedicine. communicated with ISDN and Internet to the Intranet of NTUH. Inside NTUH, we allocated four points: the telemedicine clinic room at the Department of Family Medicine, the discussion room at $6 \mathrm{~A}$ inpatient building, the telemedicine room 405 and the teleeducation room 101 at the College of Medicine. NTUH is also connected with Puli Christian Hospital (PCH) at mid-Taiwan. Meanwhile, another national hospital, National Cheng-Kung University Hospital (NCKUH) located at south Taiwan, is fully participating and connected with Cardinal Tien Hospital (CTH).

To increase the service ratio and quality of the telemedicine, we propose the Union Telemedicine Clinics Center (UTCC) concept to achieve our goal. To show the power of telemedicine services, we choose five remote tribes listed as above, because all of them lack of healthcare resources.

We design, implement and setup an Integrated Telemedicine Healthcare Equipment (ITHE). The patients who live at the remote tribes are lack of healthcare resources except for the period of the regional Circular Healthcare $(\mathrm{CH})$ services. Hence we train the nurse and volunteer how to use the ITHE system to immediately help the patients. The ITHE could be connected to doctor at the hospital site (DHS), with ADSL lines. Therefore, the UTCC and ITHE let most of the patients who live at remote tribes obtain the healthcare consulting services before they are transferred to the hospital. That is, the UTCC 
compensates the vacancy of the routine regional $\mathrm{CH}$ services within such areas.

In accordance with the Communication Operating Model (COM) which announced by the DOH-Taiwan, each remote nursing station (RNS) should at least support 55 standing drugs, an electronic healthcare record (EHR) system, a fax machine, the prescription which is ordered by DHS. Then the trained and qualified nurses or volunteers at RNS could use the prescription to treat a specific patient.

In order to establish the telemedicine clinics at tribes, the UTCC could collect all of the resources of the telemedicine clinics at DHS. The processing flow uses network to connect RNS and DHS. The patient of a specific tribe could register his favorite clinic with the assist of internet or nurses at that tribe. Then the patient could fill the basic personnel information and start diagnosis in a proper orcer. DHS will record the diagnosis at telemedicine EHR and write down the prescription. RNS will print out the current clinical data, keep it in files, and follow up DHS orders to give patient assigned medicine or therapy. Figure 1 illustrates the EHR could diagnose and prescribe by DHS under the UTCC environment.

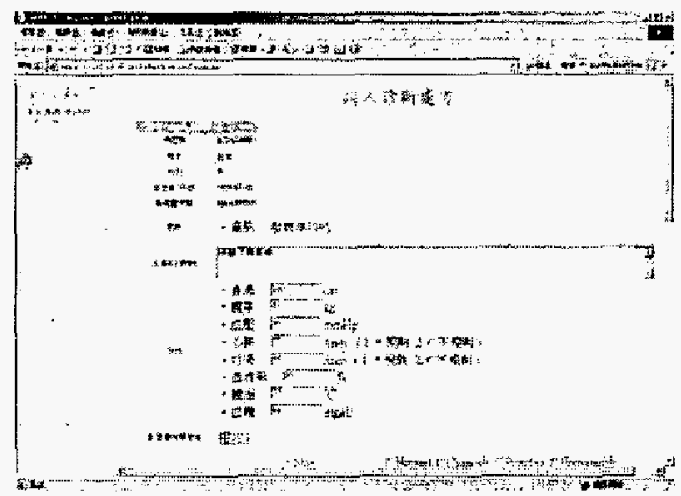

Figure 1: The EHR of UTCC

Table 1: Comparison between ITHE and EHC

\begin{tabular}{lll}
\hline Items & ITHE & EHC \\
\hline Implementation Cost & US\$4,300 & US\$8,500 \\
Maintenance Cost & US\$500 & US\$3,500 \\
Communication Mode & ADSL & ISDN \\
Vital Sign & Available & Available \\
Glucose & Available & Available \\
$\mathrm{SaO}_{2}$ & Available & Available \\
Body Temperature & Available & Available \\
Body Weight & Available & Available \\
Stethoscope & Available & Available \\
Expansible Capability & Easy & No Available \\
\hline
\end{tabular}

Another similar telephone operating interface is IP phone [13]. The advantages of this operating model are easy installation, clear screen image, and clear voice quality. Meanwhile, we could connect externally to another larger screen and zoom in/out the control camera lens. In fact, this model could act as a public education platform for health and nutrition or remote emergent communication equipment for the tribe people. The consultant contents could be the notations for diabetes, hypertension, etc.

The UTCC includes four system modules: Internet Registration Management System (IRMS), Internet Patient Registration System (IPRS), Internet Clinics System (ICS), and ITHE. Figure 2 illustrates the related functionalities of these four system modules.

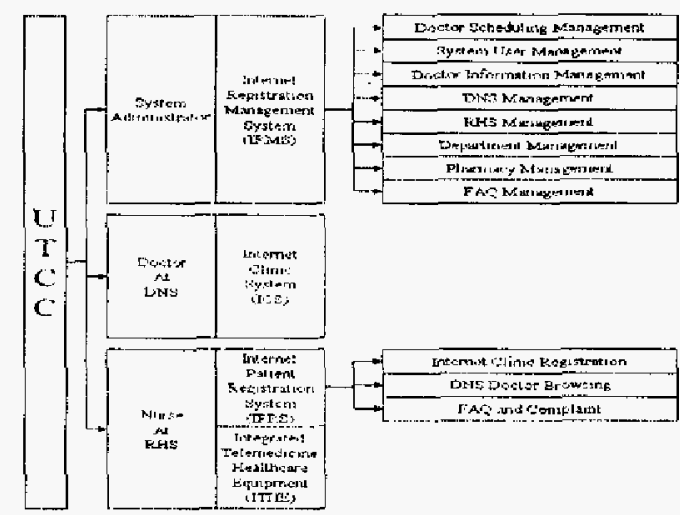

Figure 2: The related functionalities of UTCC

In order to extend our UTCC with lower limitations, we use the HL7 [14] standard to implement the data exchange interfaces inside our UTCC system.

Telemedicine doctors at DHS could depend on his clinical specialty to divide the patient types. Under the telemedicine referral duration, doctors could judge the field of the patient's condition through the screening by the nurses at RNS. Hence the doctors could concentrate the clinical appointment period and focus on the specific patient's condition to give them specific diagnoses. That is, such healthcare behavior will let patient's diagnoses and cares gradually fit the patient's condition. Figure 3 illustrates the IPRS module of UTCC and we could easily browse all of the doctors who serve from every DHS through Internet.

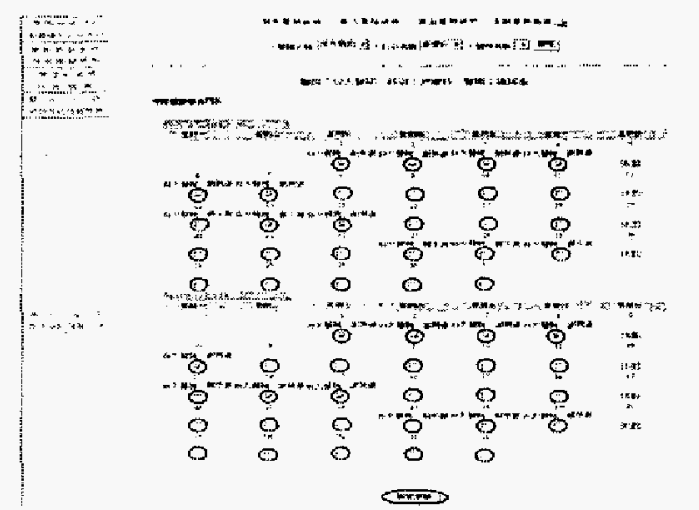


Figure 3: The IPRS module of UTCC

\section{Results}

Many failures of telemedicine system are not from technical failure. On the contrary, most of them are blindly catching up with the latest and advanced information technologies and burning their budgets. Actually, several medical referrals and consulting services could be completed with generic telephone. In fact, many generic referrals methods anong doctors are still running with such media.

An acceptable telemedicine is built on the weight between the cost and quality. We should realize our requirements and use the most feasible and effective solutions to earn the maximum fruitful results and minimum expenses. Examples of successful cases show that we could use digital camera and e-mail to achieve the dermatology clinical referral, use fax machine to complete the judgment of remote electrocardiogram, use mail to fulfill the remote radiology clinical referral of the $\mathrm{X}$ ray films [13]. All of the above cases might already prove that it is feasible to use the low cost and reasonable information technology solutions to achieve telemedicine.

Originally, the EHC-350 is used at home care environment. Hence it emphasizes on its simplified usage for everybody. Nevertheless, it is doubtable whether it is necessary to use such highly expensive integrated home care system under the assistance of nurses or professionals at each RNS.

Moreover, we frequently emphasize on the on-line, live, and interactive conference functionality. Sometimes we might ignore much useful information. Practically, some information could use a stored and forward mechanism to refer from RNS to DHS at first. For example, we could pass the vital sign, glucose, and $\mathrm{SaO}_{2}$ values which are gathered at RNS and send to DHS. Such operation only needs a simple teleconference system or a video phone to achieve the clinical refertal. Based on the current quotations on the medical market, the total price of a digital hemadynamometer, blood sugar meter, electronic stethoscope, and a teleconference system are under our acceptable budget. The above medical instruments could be operated easily by most RNS. This is a rcasonable and feasible solution and doctor referral model is illustrated as follows.

- Before the remote consulting process, RNS should gather the vital sign, glucose, and $\mathrm{SaO}_{2}$ in advance and record data by hand, then fax or e-mail to DHS.

- DHS can diagnose with data recorded by the telemedicine system.

- DHS can advice and fax prescription to RNS.
Telemedicine had been implemented for a long time and the implementation technology gradually matured. Supported by the government, medical centers, and regional hospitals, the patient who lives at remote area could be served with similar metropolitan healthcare quality. This not only conquers the spatial and temporal unavailability, but also lets the medical resources be shared with mountain tribes.

Based on this project, both NTUH and NCKUH ally each other. Horizontally, we integrated with UTCC and NTUH designed the low cost ITHE. Through the cheap IP phone, we compensate the communication quality, promote the diagnosis quality, let doctor at DHS get accurate symptom from remote patients.

The church power inside the tribes heip us to advise and educate the remote people, even act as a volunteer and help the telemedicine operating. Obviously, church helps let people like to be diagnosis. with telemedicine system and increase the usage ratio of the telemedicine equipments.

However, the scheduling appointments between the patients and doctors at DHS could not be easily made. The annual survey statistics from our five tribes, as shown in Tables 4 to 6 , will illustrate such requirements from people who live at tribes. Both surveys have seven questions and we group into two categories, four tribes of $\mathrm{PCH}$ and one tribe of CTH.

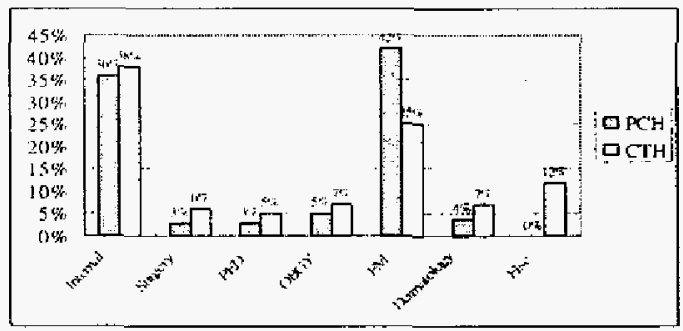

Figure 4: Survey result of the most prefer clinic

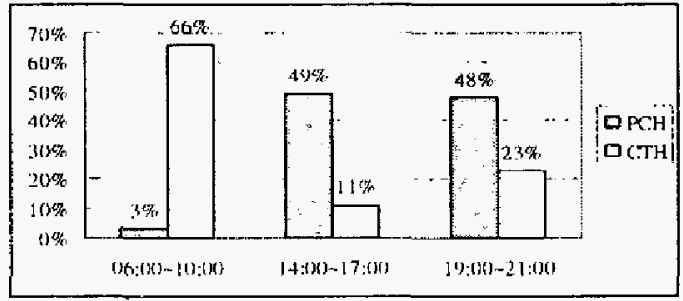

Figure 5: Survey result of the most prefer clinic time

\section{Discussion}




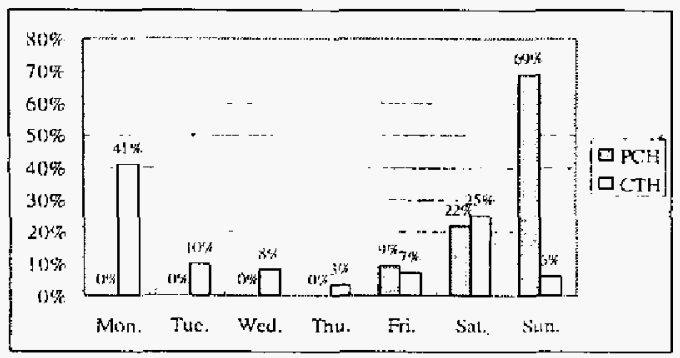

Figure 6: Survey result of the most prefer clinic day

Hopefuliy, UTCC could ally more medical centers and group more doctors to serve more tribe people. Since ITHE could gather several biomedical signals and let DHS diagnose in advance, ITHE implements a direct healthcare system which is necessary for tribe people. The IP phone could promote the telemedicine services and act as a public education platform for $\mathrm{CME}$ and remote acute communication equipment.

\section{Conclusions}

We conclude this paper with some important and feasible points as follows.

- ITHE integrates the telemedicine experience and EHR system of NTUH to serve the hilly areas.

- A UTTC is constructed to integrate the medical resources that can be shared by each hospital.

- Low cost ITHE modules are designed and deployed to tribes.

- ITHE improves the unavailability of doctors in hilly tribes by the support of diverse clinics.

- ITHE remote education system promotes the quality of continuing medical education (CME).

- Performance and feasibility of the telemedicine system have been evaluated.

\section{Acknowledgements}

This research, "Mountain and Island Telemedicine NTUH Cross-domain Telemedicine Project for Aboriginal Area," is fully supported by the DOHTaiwan and NTUH.

\section{References}

[1] Chung-Chih L., Heng-Shuen C., Ching-Yu C., AND SHENG-MOU H. (2001): 'Implementation and Evaluation of a Multimedia Telemedicine System in NTUN, Int'7 J. Med. Info, 6, pp.175-182
[2] ChUNG-CHiH L., WEN-HAO H, JYH-HORNG C., Heng-Shuen C., ChIng-Yu C., AND Sheng-Mou H. (2000): 'A Middleware Architecture to Improve the Efficiency of Web-based Telemedicine Application", Biomed. Eng. App. Basis and Commu., 20(3), pp. $/ 27-132$

[3] Chung-Chih L., Jeng-Ren D., Chien-Tasi L., HENG-SHUEN C., JENN-Lung S., AND JYH-HORng C. (1998): 'An Unified Multimedia Database System to Support Telemedicine', IEEE Trans. Infor. Technol. BioMed.. 2(3), pp. 183-192

[4] Walter H. H. (1974): 'Telemedicine: New Application of Communication Technology', IEEE Trans. Commus. pp.685-688

[5] William J. C., Robert O. R., James M. C., and RALPH M. (1997): An Architecture for Naval telemedicine:. IEEE Trans. Infor. Technol. BioMed., 1(1), pp.73-79

[6] Francesco P., Marco M., and Gabriella T. (2003): The Educational Offer in Medical Informatics and Telemedicine at Engineering Faculty of the Politeenico di Milano', IEEE Trans. Edu., 46(3), pp.394-398

[7] M. Howard W., Gavin V., George V., and DAvid M. (2001): Developing a Regional Healthcare Information Network', IEEE Trans. Infor. Technol BioMed., 5(2), pp.177-180

[8] EHSAN O. S. AND Ravi S. (2002): 'ATMTN: A Telemommography Network Architecture', IEEE Trans. BioMed. Eng, 49(12), pp.1438-1443

[9] PÉter V., ZOLTAN B., AND BALAZS B. (2002): 'An Open Architecture Patient Monitoring System Using Standard Technologies', IEEE Trans. Infor. Technol. BioMed, 6(1), pp.95-98

[10]MARINA K. (1997): 'Telemedicine: A Computer / Communications Current and Future Challenge", IEEE Potential, pp.29-31

[11]David L. P., Keri E. P., and Reuben R. M. J. (1999): Assessing Technological Barriers to Telemedicine: IEEE Trans. Eng. Mgmt., 46(3), pp. $279-288$

[12]DAVID T. M., JONATHAN S., AND WILl.IAM C. R. (2000): 'Building an IP Network Quality-of-Service Tested', IEEE Internet Comp., pp.65-73

[13] EnRIQUe J. G., Francisco D. P., Emilio J. O., Norberto M., AND Hellmer R. (1998): 'A Broadband Multimedia Collaborative System for Advanced Teleradiology and Medical Imaging Diagnosis, IEEE Trans. Infor. Technol. BioMed., 2(3), pp. 146-155

[14]HL7, Internet site address: http://www.hl7.org/ 